\title{
Concomitant Filled Prescriptions of 0xymorphone or Oxycodone with CYP3A Inhibitors and Inducers
}

\author{
D. Tyler Coyle, MD, MS; Tiffany S. Woodworth, MPH, MBA; David Moeny, RPh, MPH; \\ Judy Staffa, PhD, RPh; Tamra Meyer, PhD, MPH; Corinne Woods, RPh, MPH; Emily C. Welch, MPH; \\ Kevin Haynes, PharmD, MSCE; Sengwee Toh, ScD; and Judith C. Maro, PhD, MS
}

\begin{abstract}
BACKGROUND: 0xymorphone's metabolism does not involve the hepatic cytochrome P450 (CYP) system. The effect of this pharmacokinetic feature of oxymorphone on opioid prescribing is unknown.

OBJECTIVE: To assess the relative frequency with which oxymorphone and oxycodone (a CYP3A-metabolized opioid analgesic) were each prescribed to patients concomitantly receiving CYP3A-modifying drugs (i.e., inducers and inhibitors) to characterize opioid-prescribing patterns in patients at risk for CYP3A-related drug interactions.
\end{abstract}

METHODS: We analyzed the Sentinel Distributed Database from January 1, 2013, to December 31, 2016, to identify the proportion of patients with concomitant dispensing of selected CYP3A modifiers among initiators of oxymorphone. We then repeated the analysis using oxycodone instead of oxymorphone. We conducted sensitivity analyses that varied the washout periods for each opioid to account for potential opioid switching.

RESULTS: In the primary analysis, the proportion of patients with concomitant incident dispensings of oxymorphone and selected CYP3A modifiers was $3.26 \%(95 \% \mathrm{Cl}=3.09 \%-3.43 \%)$, and the proportion of patients with incident dispensings of oxycodone and selected CYP3A modifiers was $2.82 \%(95 \% \mathrm{Cl}=2.79 \%-2.85 \%)$. The difference between proportions was $0.43 \%(95 \% \mathrm{Cl}=0.26 \%-0.60 \%)$. Sensitivity analyses that varied the washout periods for each opioid with respect to the other opioid to account for switching yielded similar results.

CONCLUSIONS: We observed similar proportions of patients using selected CYP3A modifiers concomitantly with both oxymorphone and oxycodone. While the Cls of the point estimates did not overlap, the absolute differences between the proportions were small.

J Manag Care Spec Pharm. 2020;26(5):668-72

Copyright $\odot 2020$, Academy of Managed Care Pharmacy. All rights reserved.

\section{What is already known about this subject}

Oxymorphone's metabolism does not involve the hepatic cytochrome P450 (CYP) system, making it a potentially useful opioid analgesic for patients at risk for drug interactions.

Prescribers consider many factors when choosing particular therapeutics, including cost and side-effect profile.

\section{What this study adds}

This study assessed the relative frequency with which oxymorphone and oxycodone (a CYP3A-metabolized opioid analgesic) were each prescribed to patients receiving concomitant CYP3Amodifying drugs (i.e., inducers and inhibitors) to characterize opioid-prescribing patterns in patients at risk for selected drug interactions.

Similar proportions of patients dispensed either oxymorphone or oxycodone were also dispensed CYP3A-modifying drugs, and while the CIs of the point estimates did not overlap, the absolute differences between the proportions were small.

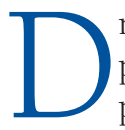
rug interactions are an important concern for patients, providers, payers, regulators, and pharmaceutical companies. Opioid-related drug interactions are notable because of the potential safety risks associated with interference in the metabolism and/or absorption of opioid products that can depress respiration. ${ }^{1}$ Many opioid analgesic-related overdose death investigations identify additional prescription drugs taken concomitantly as contributory to the fatal outcome. ${ }^{2}$

Oxymorphone is an opioid agonist indicated for the management of acute pain severe enough to require an opioid analgesic and for which alternative treatments are inadequate. Endo Pharmaceuticals submitted documents to a March 2017 Advisory Committee meeting stating that oxymorphone "may offer distinct benefits to patients who are taking multiple medications" because oxymorphone's metabolic pathway does not involve the hepatic cytochrome P450 (CYP) system. ${ }^{3}$ The CYP3A system is often involved in drug metabolism, and strong CYP3A inducers (e.g., rifampin and phenytoin) or inhibitors (e.g., ritonavir and ketoconazole) can alter systemic concentrations of CYP3A-metabolized drugs used concomitantly. CYP-related drug interactions can result in significant adverse clinical events, including myopathy, orthostatic hypotension, and drug toxicity. ${ }^{4}$ When used concomitantly with a CYP3A-metabolized opioid analgesic, a CYP3A inducer could result in inadequate pain control, whereas a CYP3A inhibitor could contribute to respiratory depression. Because oxymorphone's metabolism is through glucuronidation rather than CYP3A metabolism, use of this product, compared with 
FIGURE 1 Pictorial Summary of Analytic Episode Constructions for Primary Analysis Examining Concomitant Use of Study Opioid (Oxymorphone and Oxycodone) and CYP3A Modifiers ${ }^{\mathrm{a}}$

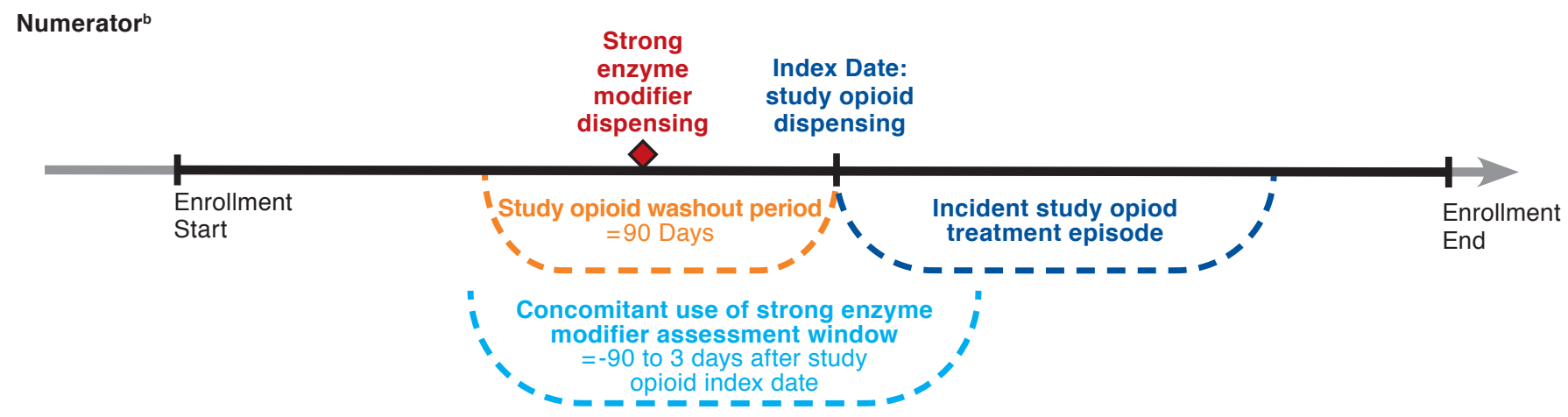

Denominator $^{\mathrm{c}}$

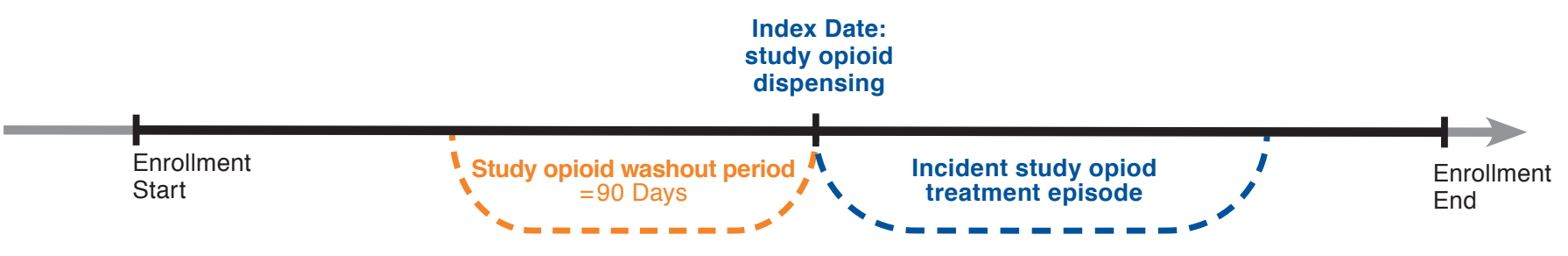

ancludes inducers and inhibitors.

${ }^{b}$ Numerator: Patients with a concomitant incident study opioid and any CYP3A modifier episode.

cDenominator: Patients with an incident study opioid episode.

CYP3A-metabolized opioid analgesics (e.g., oxycodone, hydrocodone, fentanyl, codeine, methadone, and tramadol), ,5 may therefore mitigate the risk of drug interactions in patients taking CYP3A-modifying drugs (i.e., inducers or inhibitors).

While prescribers have many considerations when choosing treatment selection, the effect of oxymorphone's metabolism on prescriber choice of opioid analgesic among patients at risk for drug interactions is unknown. We calculated the difference in proportions among oxymorphone-receiving patients who received CYP3A modifiers concomitantly and oxycodonereceiving patients who received CYP3A modifiers concomitantly. While CYP3A inhibitors are particularly relevant for this analysis, given the potential for life-threatening respiratory depression when used with opioids, we analyzed inducers as well because the undertreatment of pain is a serious problem that can significantly affect patient well-being. We hypothesized that if oxymorphone's metabolism influenced prescribing practices, we would observe a marked difference in proportions among concomitant users of each studied opioid and CYP3A-modifying drugs.

\section{Methods}

\section{Data Source}

The U.S. Food and Drug Administration (FDA)'s Sentinel Initiative is a national distributed data system designed to actively monitor the safety and effectiveness of prescription drug products in the United States. ${ }^{7}$ The 16 participating Data Partners include national and regional health insurers and integrated delivery systems. All collect administrative claims data as part of their regular business. The integrated delivery systems also provide information from electronic health records. Data were converted into a standard format, periodically updated, quality checked, and stored locally under control of the Data Partners. For this analysis, we included the data from 16 Data Partner sites from January 1, 2013, through the end of available data, which differed by Data Partner but spanned through December 31, 2016.

\section{Identification of 0xymorphone and 0xycodone Exposures}

Eligible patients must have been continuously enrolled with pharmacy benefits for at least 90 days before and including the date of their first qualifying incident exposure (i.e., the index date). We identified new users of each opioid analgesic, defined as having had no previous dispensings of the opioid of interest in the 90 days before the index date of exposure, for inclusion in the primary analysis (Figure 1). We assessed patients first valid incident dispensing only. In sensitivity analyses, we imposed additional exclusions to account for switches between the analyzed opioid analgesics, requiring new users of the 


\begin{tabular}{|c|c|c|c|c|c|c|}
\hline TABLE & $\begin{array}{l}\text { Patients with a } \\
\text { Dispensing of St } \\
\text { January 1, } 2013\end{array}$ & $\begin{array}{l}\text { Valid First Incident } \\
\text { rong CYP3A Modi } \\
\text {-December } 31,20\end{array}$ & $\begin{array}{l}\text { thispensing of Ox } \\
\text { lifiera: } 16 \text { Partners in } \\
016\end{array}$ & $\begin{array}{l}\text { phone or O } \\
\text { Sentinel Di }\end{array}$ & $\begin{array}{l}\text { ycodone and } \\
\text { ibuted Datat }\end{array}$ & comitant \\
\hline & $\begin{array}{c}\text { Patients with } \\
\text { Concomitant Opioid } \\
\text { and CYP3A Modifier } \\
\text { Episode (n) }\end{array}$ & $\begin{array}{l}\text { Patients with Opioid } \\
\text { Episodes (n) }\end{array}$ & $\begin{array}{c}\text { Patients with } \\
\text { Concomitant } \\
\text { Episodes/Patients with } \\
\text { Opioid Episodes (\%) }\end{array}$ & $95 \% \mathrm{CI}$ & $\begin{array}{c}\Delta \text { Between } \\
\text { Proportions (\%) }\end{array}$ & $\begin{array}{c}95 \% \text { CI for } \\
\Delta \text { Between } \\
\text { Proportions } \\
\end{array}$ \\
\hline \multicolumn{7}{|l|}{ Primary analysis } \\
\hline Oxymorphone & 1,400 & 43,009 & 3.26 & $3.09-3.43$ & \multirow{2}{*}{0.43} & \multirow{2}{*}{$0.26-0.60$} \\
\hline Oxycodone & 44,423 & $1,574,505$ & 2.82 & $2.79-2.85$ & & \\
\hline \multicolumn{7}{|c|}{ Sensitivity analysis 1} \\
\hline \multirow[t]{2}{*}{ Oxymorphone } & Without history of oxyc & odone use any time befor & re oxymorphone index dat & 3 days thereafter & \multirow{2}{*}{0.43} & \multirow{2}{*}{$0.17-0.70$} \\
\hline & 567 & 17,601 & 3.22 & $2.96-3.48$ & & \\
\hline \multirow[t]{2}{*}{ Oxycodone } & \multicolumn{4}{|c|}{ Without history of oxymorphone use any time before oxycodone index date and 3 days thereafter } & & \\
\hline & 43,119 & $1,547,393$ & 2.79 & $2.76-2.82$ & & \\
\hline \multicolumn{7}{|c|}{ Sensitivity analysis 2} \\
\hline \multirow[t]{2}{*}{ Oxymorphone } & Without & history of oxycodone use & se during entire enrollment & & \multirow{2}{*}{0.61} & \multirow{2}{*}{$0.30-0.92$} \\
\hline & 458 & 13,514 & 3.39 & $3.08-3.80$ & & \\
\hline \multirow[t]{2}{*}{ Oxycodone } & \multicolumn{4}{|c|}{ Without history of oxymorphone use during entire enrollment period } & & \\
\hline & 42,938 & $1,545,379$ & 2.78 & $2.75-2.81$ & & \\
\hline
\end{tabular}

drug of interest to have no recorded dispensings of the other study drug in their available enrollment history. We included all oral solid formulations of oxymorphone and oxycodone in our analysis, including immediate- and extended-release formulations, and identified dispensings using National Drug Code (NDC) numbers from outpatient pharmacy claims. We used oxycodone as a comparator opioid analgesic because it is metabolized by the CYP3A system and is widely prescribed.

\section{Identification of CYP3A Inhibitors and Inducers}

We identified the following CYP3A inhibitors using NDC numbers from outpatient pharmacy dispensings: boceprevir, cobicistat, elvitegravir, indinavir, itraconazole, ketoconazole, lopinavir/ritonavir, ombitasvir/paritaprevir/ritonavir, ombitasvir/paritaprevir/ritonavir/dasabuvir, posaconazole, ritonavir, saquinavir, telaprevir, tipranavir, and voriconazole. We identified the following CYP3A inducers in the same manner: carbamazepine, enzalutamide, mitotane, phenytoin, and rifampin. We chose these CYP3A inhibitors and inducers based on their strong CYP3A-modifying characteristics. ${ }^{4,8}$ We required that the dispensing of CYP3A modifiers occur in the -90 to +3 days surrounding the date of the opioid analgesic dispensing. This was to account for simultaneously written prescriptions filled on separate days because of differential pharmacy inventory of the products.

\section{Analysis}

Concomitancy was defined as the dispensing of a CYP3A modifier in the -90- to +3-day window surrounding the index date of a study opioid analgesic prescription. We calculated the proportion and 95\% confidence interval (CI) of all incident opioid analgesic users who had concomitant CYP3A-metabolized drug prescriptions in that window. We identified the proportion of patients with incident dispensings of oxymorphone and any dispensings of selected CYP3A modifiers in the window among all patients with an incident dispensing of oxymorphone. We then calculated the proportion of patients with incident dispensings of oxycodone and any dispensings of these selected CYP3A modifiers in the window among all patients with an incident dispensing of oxycodone and calculated differences between the groups' proportions. We created the analytic datasets using the Sentinel Cohort Identification and Descriptive Analysis Tool version 4.0.0.

In sensitivity analyses, we varied the washout periods (i.e., the time from last filled prescription) for each opioid analgesic analyzed with respect to the other opioid analgesic to account for patients switching between the 2 analyzed opioid analgesic products. The sensitivity analyses were performed to account for patients who switched between the studied products, since frequent switching could make the findings of the primary analysis difficult to interpret.

Sensitivity analyses and the primary analysis used identical lookback periods. Sentinel is a public health surveillance activity that is not under the purview of institutional review boards.

\section{Results}

\section{Primary Analysis}

Among patients with incident oxymorphone use, the proportion of patients with incident dispensings of oxymorphone and 
any dispensings of selected strong CYP3A modifiers was 3.26\% (95\% CI =3.09\%-3.43\%; Table 1). Among patients with incident oxycodone use, the proportion of patients with incident dispensings of oxycodone and any dispensings of selected strong CYP3A modifiers was 2.82\% (95\% CI=2.79\%-2.85\%). The CIs for the point estimates calculated in the primary analysis did not overlap. The difference between proportions was $0.43 \%$ (95\% CI $=0.26 \%-0.60 \%)$.

\section{Sensitivity Analyses}

Among patients without history of oxycodone use at any time in their enrollment history before the oxymorphone index date and 3 days thereafter, the proportion of patients with incident dispensings of oxymorphone and any dispensings of selected strong CYP3A modifiers was 3.22\% (95\% CI $=2.96 \%-3.48 \%$ ). Among patients without history of oxymorphone use at any time in their enrollment history before the oxycodone index date and 3 days thereafter, the proportion of patients with incident dispensings of oxycodone and any dispensings of selected strong CYP3A modifiers was 2.79\% (95\% CI $=2.76 \%$ $2.82 \%)$. The difference between proportions was $0.43 \%$ (95\% $\mathrm{CI}=0.17 \%-0.70 \%)$.

Among patients without observation of oxycodone exposure in their entire observation period preceding and following the oxymorphone index date, the proportion of patients with incident dispensings of oxymorphone and any dispensings of selected strong CYP3A modifiers was 3.39\% (95\% CI =3.08\%$3.80 \%$ ). Among patients without observation of oxymorphone exposure in their entire observation period preceding and following the oxycodone index date, the proportion of patients with incident dispensings of oxycodone and any dispensings of selected strong CYP3A modifiers was 2.78\% (95\% CI $=2.75 \%$ $2.81 \%$ ). The difference between proportions was $0.61 \%$ (95\% $\mathrm{CI}=0.30 \%-0.92 \%)$. The CIs for the point estimates calculated in the sensitivity analyses did not overlap.

\section{Discussion}

The proportions of oxymorphone-CYP3A modifier concomitancy and oxycodone-CYP3A modifier concomitancy were similar, with small $(<1 \%)$ proportional differences between groups in all scenarios. While the CIs of the point estimates did not overlap, the absolute differences between the proportions were small. These results suggest that oxycodone's and oxymorphone's metabolism have a limited effect on prescribing practices, since clinicians appeared to prescribe either opioid analgesic concomitantly with CYP3A modifiers despite the potential benefit of oxymorphone's metabolism not involving CYP enzymes. While recognizing that many factors influence opioid treatment selection, this analysis suggests that there may be thousands of patients using CYP3A modifiers who were prescribed oxycodone but might potentially benefit from being prescribed oxymorphone instead.
Prescription opioid analgesics have been the subject of increased public health attention in the past decade because of increasing rates of opioid-related misuse, abuse, overdose, and death. ${ }^{9}$ Opioid-related drug interactions are particularly concerning because many opioid analgesic-related overdose death investigations identify additional prescription drugs taken concomitantly as contributory to the fatal outcome. ${ }^{2}$ Oxycodone is an appropriate reference group for this analysis because both molecules were schedule II opioids for the entire study period, unlike hydrocodone, which is important because scheduling may affect prescribing practices. ${ }^{10}$ That said, oxycodone and oxymorphone have key differences beyond their metabolic pathways. Oxycodone's morphine milligram equivalent (MME) factor is 1.5, whereas oxymorphone's MME is 3, thus, oxymorphone is approximately twice as potent on a milligramto-milligram basis as oxycodone. ${ }^{11}$ Data on real-world opioid analgesic prescribing practices are essential to inform stakeholders about how these products are being used and to guide drug interaction risk reduction strategies in patients with pain severe enough to require an opioid analgesic.

\section{Limitations}

This analysis has limitations. First, the analysis offered no insight into the provider's rationale for the opioid analgesic selected, making any inferences about drivers of clinical prescribing practices speculative. Second, the analysis did not adjust for individual patient characteristics and comorbidities that could affect pharmacotherapy selection, and there may be key clinical differences among patients treated with oxymorphone compared with patients treated with oxycodone. Third, the analysis did not account for MME, duration of therapy, or other potentially relevant utilization metrics that could meaningfully inform the relationship between the analyzed opioid analgesics and CYP3A modifiers. Fourth, a 90-day window was chosen to identify concomitancy because many CYP3A modifiers may be dispensed with a 90-day supply. This window may overestimate concomitancy, especially if patients are nonadherent. Finally, Sentinel only captures dispensed medications that are paid for by insurance; therefore, any prescriptions paid for with cash are not captured in the data. In addition, the data source provides no information about prescription cost or copays, which could also have been a factor that affected product choice.

\section{Conclusions}

Similar proportions of patients dispensed either oxymorphone or oxycodone were also dispensed CYP3A modifiers concomitantly. While the CIs of the point estimates did not overlap, the absolute differences between the proportions were small. We found little evidence to support the hypothesis that oxymorphone's metabolism influenced prescriber behavior. 


\section{Authors}

D. TYLER COYLE, MD, MS; DAVID MOENY, RPh, MPH; JUDY STAFFA, PhD, RPh; TAMRA MEYER, PhD, MPH; and CORINNE WOODS, RPh, MPH, Office of Surveillance and Epidemiology, Center for Drug Evaluation and Research, U.S. Food and Drug Administration, Silver Spring, Maryland. TIFFANY S. WOODWORTH, MPH, MBA; EMILY C. WELCH, MPH; SENGWEE TOH, ScD; and JUDITH C. MARO, PhD, MS, Department of Population Medicine, Harvard Medical School and Harvard Pilgrim Health Care Institute, Boston, Massachusetts. KEVIN HAYNES, PharmD, MSCE, HealthCore, Wilmington, Delaware.

AUTHOR CORRESPONDENCE: Judith C. Maro, PhD, MS, Department of Population Medicine, Harvard Pilgrim Health Care Institute, Harvard Medical School, 401 Park Dr., Ste. 401, Boston, MA 02215. Tel.: 617.867.4494; E-mail: judy_maro@harvardpilgrim.org.

\section{DISCLOSURES}

This project was supported by Task Order HHSF22301001T under Master Agreement HHSF223201400030I from the U.S. Food and Drug Administration (FDA). The FDA approved the study protocol, including the statistical analysis plan, and reviewed and approved the manuscript. Coauthors from the FDA participated in the results interpretation and in the preparation and decision to submit the manuscript for publication. Coyle, Money, Staffa, Meyer, and Woods are employed by the FDA. The other authors have no financial conflicts of interest to report. The views expressed are those of the authors and not necessarily those of the U.S. Department of Health and Human Services, U.S. Food and Drug Administration.

\section{ACKNOWLEDGMENTS}

The authors thank the data partners who provided data used in the analysis: Aetna, Blue Bell, PA; Blue Cross Blue Shield of Massachusetts, Boston, MA; Harvard Pilgrim Health Care Institute, Boston, MA; HealthCore, Translational Research for Affordability and Quality, Alexandria, VA; HealthPartners Institute, Minneapolis, MN; Humana, Comprehensive Health Insights, Miramar, FL; Kaiser Permanente Colorado Institute for Health Research, Denver, CO; Kaiser Permanente Center for Health Research Hawai'i, Honolulu, HI; Kaiser Permanente Mid-Atlantic States, Mid-Atlantic Permanente Research Institute, Rockville, MD; Kaiser Permanente Northern California, Division of Research, Oakland, CA; Kaiser Permanente Northwest Center for Health Research, Portland, OR; Kaiser Permanente Washington Health Research Institute, Seattle, WA; Marshfield Clinic Research Institute, Marshfield, WI; Meyers Primary Care Institute, Worcester, MA; OptumInsight
Life Sciences, Boston, MA; and Vanderbilt University Medical Center, Department of Health Policy, Nashville, TN, through the Tennessee Division of TennCare of the Department of Finance and Administration, which provided data. The authors also acknowledge the contribution of Genna Panucci for her support in quality assurance procedures.

\section{REFERENCES}

1. Tarumi Y, Pereira J, Watanabe S. Methadone and fluconazole: respiratory depression by drug interaction. J Pain Symptom Manage. 2002;23(2):148-53.

2. Jones C, Mack K, Paulozzi J. Pharmaceutical overdose deaths, United States, 2010. JAMA. 2010; 309(7):657-59.

3. Endo Pharmaceuticals. Opana ER (oxymorphone hydrochloride) extended-release tablets. Joint Meeting of the Anesthetic and Analgesic Drug Products Advisory Committee and the Drug Safety and Risk Management Advisory Committee. February 3, 2017. Available at: https://www.fda.gov/ downloads/AdvisoryCommittees/CommitteesMeetingMaterials/Drugs/ AnestheticAndAnalgesicDrugProductsAdvisoryCommittee/UCM545762.pdf Accessed April 4, 2020

4. Flockhart DA. Drug interactions: Cytochrome P450 drug interaction table. Indiana University School of Medicine. 2007. Available at: http://medicine. iupui.edu/clinpharm/ddis/. Accessed April 4, 2020.

5. Lynch T, Price A. The effect of cytochrome P450 metabolism on drug response, interactions, and adverse effects. Am Fam Physician. 2007;76(3):391-96.

6. Gudin J. Opioid therapies and cytochrome P450 interactions. J Pain Symptom Manage. 2012;44(6 Suppl):S4-14.

7. Ball R, Robb M, Anderson S, Dal Pan G. The FDA's Sentinel Initiative-a comprehensive approach to medical product surveillance. Clin Pharmacol Ther. 2016;99(3):265-68.

8. U.S. Food and Drug Administration. Drug development and drug interactions: table of substrates, inhibitors, and inducers. 2016. Available at: https:// www.fda.gov/drugs/developmentapprovalprocess/developmentresources/druginteractionslabeling/ucm093664.htm. Accessed April 4, 2020.

9. Centers for Disease Control and Prevention. Prescription opioid overdose data. 2017. Available at: https://www.cdc.gov/drugoverdose/data/overdose. html. Accessed April 4, 2020.

10. Raji M, Kuo Y, Adhikari D, Baillargeon J, Goodwin J. Decline in opioid prescribing after federal rescheduling of hydrocodone products. Pharmacoepidemiol Drug Saf. 2018;27(5):513-19.

11. Centers for Medicare \& Medicaid Services. Opioid oral morphine equivalent (MME) conversion factors. 2017. Available at: https://www.cms. gov/Medicare/Prescription-Drug-Coverage/PrescriptionDrugCovContra/ Downloads/Opioid-Morphine-EQ-Conversion-Factors-Aug-2017.pdf. Accessed April 4, 2020. 